
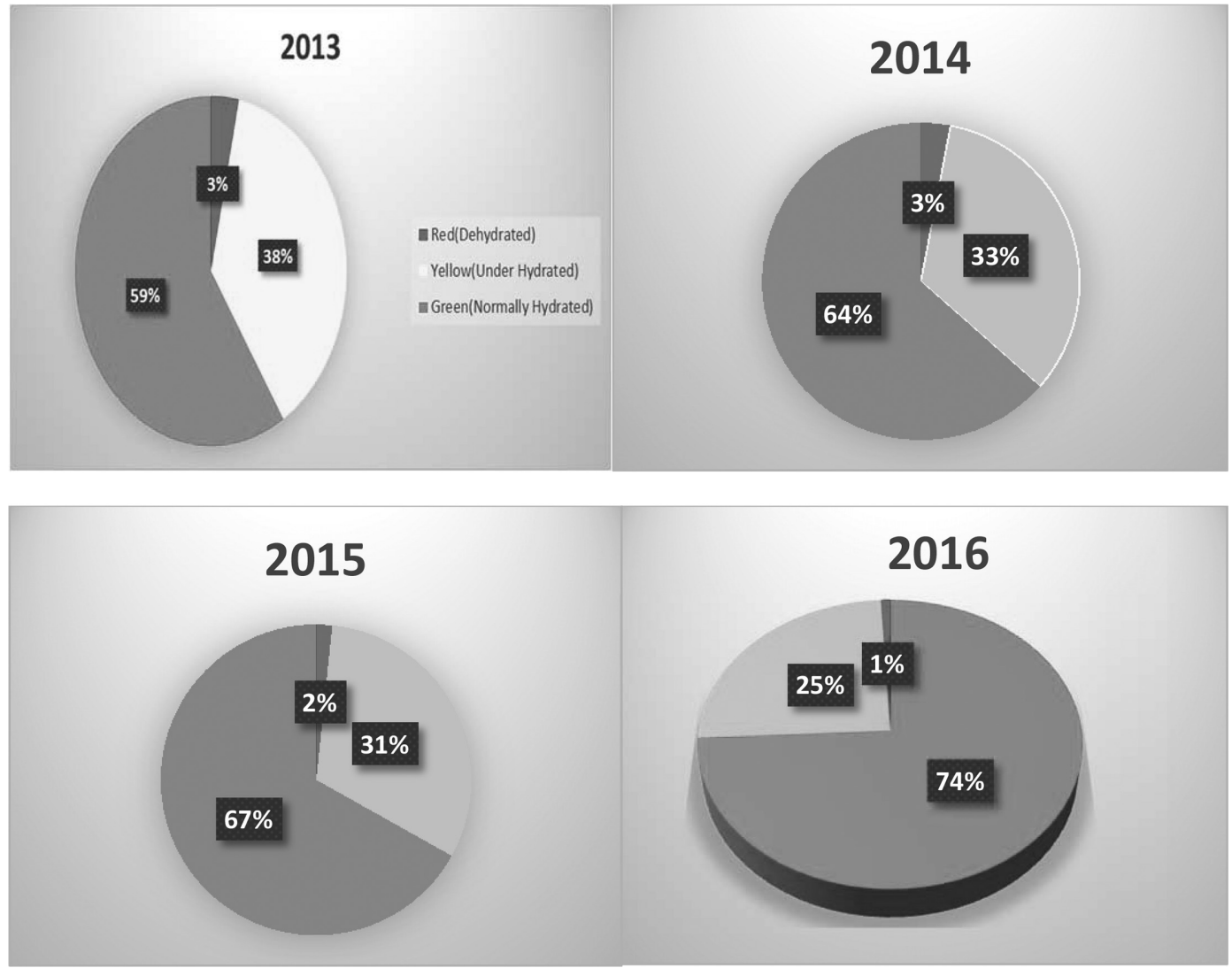

Abstract 764 Figure 1 Hydration status in tested population 2013, 2014, 2105 \& 2016 (in\%)

Results of urine specific gravity testing were analysed daily, weekly and monthly and shared with relevant leaders in management as well. Work place measurements of thermal index parameters like temperature, humidity etc. were analysed which helped in creation of 'Heat Stress Risk Map' for specific work areas and the entire organisation. These measures helped area managers to plan work activities prudently during summer months. Statistical Analyses results on the number of green cards, yellow cards and red cards were incorporated into management lean Board discussions.

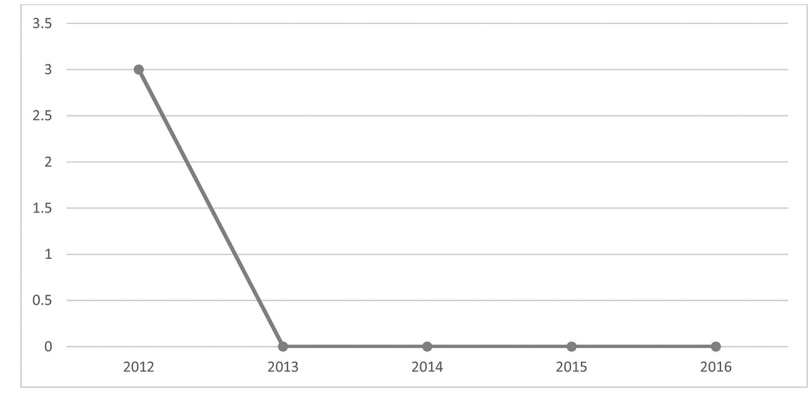

Abstract 764 Figure 2 Recordable heat illness cases (2013-2016)

Conclusion These measures among other supportive measures resulted in significant reduction of cases of heat illness, particularly elimination of recordable heat illness cases from 2013 through 2016 (figures 1 and 2).

\section{COMMUNICATING WORKPLACE HEALTH \& WELLBEING IN THE HEALTH SERVICE EXECUTIVE (HSE)}

${ }^{1}$ Emer Carroll, ${ }^{2}$ Nodlaig Carroll, ${ }^{1}$ Noelle Dalton, ${ }^{1}$ Fiona Dardis, ${ }^{3}$ Sally Downing. ${ }^{1}$ National Health and Safety Function (NHSF), Health Service Executive (HSE); ${ }^{2}$ Organizational Psychology Unit, Health Service Executive (HSE); ${ }^{3}$ National Communications Division, Health Service Executive (HSE)

\subsection{6/oemed-2018-ICOHabstracts.225}

Introduction The Health Service Executive (HSE) recognise health and wellbeing as a continuum of care for staff throughout their working life. The Workplace Health and Wellbeing (WHW) Division, established in May 2016, aims to raise awareness on the importance of safeguarding health and wellbeing at work by providing supports, tools and information that encourage a healthy working life.

To achieve this goal, a multidisciplinary project team developed an effective communication framework highlighting the support services and initiatives available through the services of Health and Safety, Occupational Health, Employee Assistance and Counselling Services and Health Promotion and Improvement, under the WHW Division.

Methods The project team reviewed international and national communication practices for health and wellbeing within the workplace. Throughout the project, the team engaged with key stakeholders and employees to identify what information staff required and how this information should be collated and governed. Using the HSE Change Management framework, agreement was reached on website design, content and promotion. 
Result The outcomes of the project included:

- the establishment of an online presence for members of WHW and HSE staff

- a sustainable communications framework that provides relevant and up-to-date

Information (website/pamphlet):

- the foundation for a Community of Practice within WHW to aid future learning

- a governance structure for future WHW communications

- the collation of information on the WHW services and a communications plan for launch of resources

Discussion A key factor in the success of this project was identifying the resources required to meet the needs of our target audience and in the establishment of 'networks' to ensure the continuous development of these resources. We foresee this project to be very extremely positive as it will enhance staff engagement with workplace health and wellbeing into the future.

\section{CONTEXTUALISED KNOWLEDGE SYNTHESIS FOR LOCAL STAKEHOLDERS IN OHS}

${ }^{1}$ Emma Irvin*, ${ }^{2}$ Stephen Bornstein, ${ }^{1}$ Kim Cullen, ${ }^{2}$ Amanda Butt, ${ }^{1}$ Dwayne Van Eerd, ${ }^{3}$ Leslie Johnson, ${ }^{3}$ Steven Passmore, ${ }^{1}$ Ron Saunders. 'Institute for Work and Health, Toronto, Canada; ${ }^{2}$ SafetyNet, Memorial University, St Johns, Canada; ${ }^{3}$ University of Manitoba, Winnipeg, Canada

\subsection{6/oemed-2018-ICOHabstracts.226}

Introduction Effective decision-making in occupational health and safety (OHS) requires having up-to-date evidence on what works from the best available research. However, the research literature does not typically take into account how implementation may be constrained by the demographic, economic or resource context of a jurisdiction/region that is considering action. Evidence-informed practices and policies need to be made based not only on an understanding of 'what works', but also on an understanding of 'what will work here'. Our objective was to develop and test an innovative methodology for synthesising and contextualising current scientific knowledge in occupational health and safety.

Methods The teams combined methods used by the 'Contextualised Health Research Synthesis Program' (CHRSP) at the Newfoundland and Labrador Centre for Applied Health Research (NLCAHR) with techniques for systematic reviewing and reporting pioneered by the Systematic Review Program at IWH. In our pilot testing we collected data about important contextual factors from key stakeholders through interviews or focus groups.

Result The method we developed describes a variety of synthesis methods that can be used by researchers and stakeholders for evidence-based decision-making. In addition, we describe the process of gathering contextual information from key stakeholders. In one example related to depression in the workplace, we found that contextual factors of geography, industry/workplace, safey culture were important for stakeholders to consider in implementing evidence-based interventions.

Discussion The project resulted in the creation of Evidence in Context Occupational Health and Safety Operational Handbook and an updated systematic review Managing Depression in the Workplace that was contextualised for the province of Manitoba (http://www.iwh.on.ca/systematic-reviews). The methodology has been transferred to end users in three Canadian provinces to date, Newfoundland and Labrador, Ontario and Manitoba. The handbook is a practical and relatively inexpensive way for OHS stakeholders to synthesise and contextualise evidence for decision making.

\section{Construction Occupational Safety and Health}

\section{CONSTRUCTION SAFETY \& HEALTH IN EMERGING ECONOMIES: DEVELOPING BEST PRACTICES WITH RESOURCE CONSTRAINTS}

Knut Ringen*. CPWR, Maryland, USA

10.1136/oemed-2018-ICOHabstracts.227

Aim of special session To share experience of improvement efforts in the subject areas where resources are limited

${ }^{1}$ Ramana KR, ${ }^{2}$ Somnath Ganguly, ${ }^{3}$ Krishna Nirmalya Sen, ${ }^{4}$ Preety Gupta, ${ }^{5}$ Arijit Chatterjee

${ }^{1}$ National Academy of Construction, Hyderabad, India

${ }^{2}$ Calcutta University, Kolkata, India

${ }^{3}$ Larsen \& Toubro Limited, Kolkata, India

${ }^{4}$ Independent Consultant, Goa, India

${ }^{5}$ Kalyani University, Kalyani, India

\section{$1657 a$ SKILLING HELPS IN IMPROVING PRODUCTIVITY, QUALITY AND SAFETY IMPLEMENTATION AT SITE - A STUDY ON SYMPTOMS AND CONTROL MEASURES}

${ }^{1}$ KR Ramana, ${ }^{2} \mathrm{KN}$ Sen. ${ }^{1}$ Advisor, National Academy of Construction, Hyderabad, India; ${ }^{2}$ President, ASSE India Chapter, India

\subsection{6/oemed-2018-ICOHabstracts.228}

Introduction Various studies have shown that there exists huge gap between demand and supply of skilled workmen at construction sites in India. This gap has high influencing effects on productivity, quality and safety. At the level of central government, immense thrust is given to improve the skill level and skill density across the sectors, through various initiatives. At enterprise level, few leading organisations had been focussing on skill development as a part of their corporate social responsibility and business strategy.

National Academy of Construction located at Hyderabad has been playing a key role in the concerned areas.

Methods Study conducted involving participants, mainly crew members deployed at sites, after completion of skill development programs. In comparison with select control groups, performance outcome of these teams under study in terms of safety, quality productivity were evaluated over a period of 6 month time, spanning participants from 52 batches.

Results Strong association with skill training and desired outcome on productivity quality safety was indicated.

Conclusion The study establishes the importance of continued and enhanced progress in the arena of construction skill development to muster the objective of sustainable growth and overall improvement in $\mathrm{H}$ and $\mathrm{S}$ performance. This gets well dovetailed with the growing economic activities, increased demands of skilled crew for construction projects across the length and breadth of the country. 